\title{
Varicocele e volume testicular em adolescentes e adultos jovens portadores de esquistossomose hepatoesplênica cirúrgica ${ }^{1}$
}

\author{
Frederico Teixeira Brandt ${ }^{2}$ \\ Carla Daisy Costa Albuquerque ${ }^{3}$ \\ Carlos Teixeira Brandt ${ }^{4}$ \\ Carolina Duarte Barbosa ${ }^{5}$
}

\begin{abstract}
Brandt FT, Albuquerque CDC, Brandt CT, Barbosa CD. Varicocele e volume testicular em adolescentes e adultos jovens portadores de esquistossomose hepatoesplênica cirúrgica. Acta Cir Bras [serial online] 2003 Jul-Ago;18(4). Disponível em URL: http://www.scielo.br/acb.
\end{abstract}

RESUMO - Objetivo: Avaliar o volume testicular e a prevalência de varicocele em adolescentes e adultos jovens portadores de esquistossomose na forma hepatoesplênica associada a varizes sangrentas de esôfago. Métodos: O estudo envolveu 22 adolescentes masculinos com esquistossomose na forma descrita, submetidos à esplenectomia, ligadura da veia gástrica esquerda e auto-implante de tecido esplênico no grande omento quando crianças. O tempo médio de seguimento foi de 4 anos após a intervenção. O grupo de voluntários sadios foi de adolescentes de uma escola na mesma região onde os casos operados viviam. Eles foram selecionados de forma randomizada, considerando a faixa de idade, características epidemiológicas e não serem infestados por equistossomose. Todos os participantes possuíam história clínica completa e foram submetidos a exame físico e ultra-sonográfico. Foi dado atenção especial as características sexuais, ultrasonografia testicular e dopplerfluxometria das veias espermáticas. Resultados: Dos pacientes classificados como adultos pela idade cronológica, 53,8\% tinham genitais com características infantis. A incidência de varicocele foi igual a $61,5 \%$ no grupo afetado e $40 \%$ no grupo controle, sem diferença estatística entre os grupos (x $2=1,77$; g.1.=1; p=0,18 3 p Fisher=0,16), acometendo predominantemente o testículo esquerdo, em ambos os grupos. Conclusão: Pacientes com doença esquistossomótica cirúrgica tinham significante déficit no desenvolvimento puberal e genital, no volume testicular, especialmente do lado esquerdo, e alta prevalência de varicocele (61.5\%) sem uma associação direta com atrofia testicular.

DESCRITORES - Varicocele.Volume testicular. Esquistossomose mansônica. Criança. Adolescente. Adulto jovem. Hipertensão porta.

\section{Introdução}

Esquistossomose mansônica na forma hepatoesplênica persiste como a causa mais comum de hipertensão porta na adolescência e em adultos jovens na região Nordeste do Brasil ${ }^{1}$. O tratamento cirúrgico associando esplenectomia e ligadura da veia gástrica esquerda nos casos que se associam a varizes sangrentas do esôfago, além de esclerose endoscópica nos casos de recidiva hemorrágica, tem produzido bons

1. Trabalho realizado nos Serviços de Urologia e Cirurgia Pediátrica da Universidade Federal de Pernambuco (UFPE). Resumo de Tese de Doutorado da UFPE.

2. Prof. Adjunto de Urologia da UFPE, Doutor e Livre Docente em Urologia pela Universidade de São Paulo (USP). Prof. Orientador da Tese.

3. Profa. Adjunta de Obstetrícia da UFPE.

4. Prof. Titular de Cirurgia Pediátrica da UFPE.

5. Acadêmica de Medicina, Bolsista de Iniciação Científica/CNPq.

342 - Acta Cirúrgica Brasileira - Vol 18 (4) 2003 
resultados. A adição de auto-implante de tecido esplênico no omento maior fez desaparecer a mortalidade por septicemia fulminante pós-esplenectomia, possibilitando maior sobrevida e permitindo a observação prolongada de crianças tratadas cirurgicamente, em idade precoce ${ }^{25}$. Nesse sentido, além dos sistemas imunológico e digestivo, outros estão sendo investigados. Entre eles o sistema urinário ${ }^{6}$.

Entre as consequiências hemodinâmicas da hipertensão porta está o estabelecimento de colaterais portosistêmicas com o desenvolvimento de dilatações varicosas do plexo venoso gastroesofageano e hemorroidário. Em alguns casos, pode haver veias dilatadas tortuosas anastomosando a veia esplênica, ou porta, com as veias renais, o que aumenta a pressão venosa nessas veias predispondo a um incremento da prevalência de varicocele $\mathrm{e}^{7,8,9}$.

Varicocele ocorre em cerca de $15 \%$ da população adulta masculina, entretanto, é pouco comum antes da puberdade. Esta doença tem sido reconhecida como uma das causas mais comuns de sub-fertilidade, ou infertilidade, estando associada à redução do volume testicular e oligoespermia ${ }^{10,11}$.

Partimos da idéia que adolescentes e adultos jovens com hipertensão porta poderia representar um modelo propício para desencadear varicocele e atrofia testicular. Desta forma, a presente investigação teve como objetivo estudar tais alterações morfológicas em portadores de esquistossomose na forma hepatoesplênica associada a varizes sangrentas de esôfago, submetidas, quando crianças, a esplenectomia, ligadura da veia gástrica esquerda e auto-implante de tecido esplênico no omento maior após seguimento médio, pós-tratamento, de quatro anos.

\section{Métodos}

Vinte e duas crianças, adolescentes e adultos jovens, do sexo masculino, de idades entre 10 e 21 anos, portadores de esquistossomose na forma hepatoesplênica grave, que tinham sido submetidos, quando crianças (idades entre oito e dezesseis anos), a esplenectomia, ligadura da veia gástrica esquerda e autoimplante de tecido esplênico no omento maior, foram incluídos na presente investigação. Todos os pacientes foram acompanhados no Serviço de Cirurgia Geral da Criança e de Urologia do Hospital das Clínicas - Centro de Ciências da Saúde, CCS - UFPE, apresentando um seguimento de um a oito anos (média de quatro anos) após o tratamento cirúrgico.

Para análise da avaliação do volume testicular, foram excluídos as crianças com idade inferior a 14 anos e aquelas com estadiamento I na escala de Tanner para desenvolvimento puberal.

Quinze adolescentes sadios, voluntários, não esquistossomóticos, na mesma faixa etária e com as mesmas características epidemiológicas, foram avaliados de forma idêntica como grupo de controle.

\section{Procedimentos}

No exame físico, deu-se ênfase particular ao exame da genitália, dos caracteres sexuais secundários, assim como do grau de desenvolvimento puberal, classificando-as segundo os critérios de Tanner. Procurou-se avaliar, à palpação, o volume testicular e possível presença de enovelado varicoso do plexo pampiniforme no cordão espermático.

A dopplerfluxometria colorida foi usada para avaliar o volume testicular, o calibre das veias do plexo pampiniforme e a pesquisa de refluxo venoso nessas veias. Foi utilizado o seguinte equipamento: Unidade de tempo real Aloka 1700 com transdutor de freqüência variável de 7 a 9-MHz.

A determinação do volume testicular foi feita utilizando-se a fórmula para determinação do volume de uma figura elipsóide:

\section{$0,523 \times$ comprimento em $\mathrm{cm} \times$ largura $\mathrm{em} \mathrm{cm}$ $x$ espessura em $\mathrm{cm}^{12}$}

A massa testicular dos pacientes foi obtida somando-se os volumes dos testículos direito e esquerdo.

A análise da diferenças entre as médias dos volumes e das massas testiculares foi realizada utilizandose o teste " $t$ " de Student para amostras não pareadas. Aceitou-se o limite de $\mathrm{p}>0,05$ para rejeição da hipótese de nulidade.

\section{Resultados}

Pela classificação de Tanner, se pode identificar retardo de desenvolvimento genital apenas no grupo afetado. Visto que 53,8\% dos pacientes classificados como adultos pela idade cronológica, tinham genitais com características infantis.

Os volumes elipsóides médios do grupo afetado foram significativamente menores que os ipso-laterais do grupo controle $(F=9,70$ e $p=0,00 ; F=10,22$ e $p=0,00$, respectivamente para testículo direito, esquerdo e volume médio).

Da análise do índice de atrofia testicular, calculado com base no volume testicular elipsóide, identificou-se que os grupos não diferiam entre si $(\mathrm{F}=1 ; \mathrm{p}=0,29)$, Tabela 1. 
Brandt F T e col.

TABELA 1 - Médias, desvio-padrão e intervalo de variação dos parâmetros testiculares em pacientes com esquistosomose mânsonica e em grupo controle.

\begin{tabular}{|c|c|c|c|c|c|c|}
\hline \multirow{2}{*}{$\begin{array}{l}\text { PARÂMETROS } \\
\text { TESTICULARES }\end{array}$} & \multicolumn{5}{|c|}{ MÉDIAS E DESVIOS-PADRÃO DOS VALORESAMOSTRAIS } & \multirow[b]{2}{*}{ Variação } \\
\hline & Direito & Variação & Esquerdo & Variação & médio & \\
\hline \multicolumn{7}{|l|}{ GRUPOAFETADO } \\
\hline $\begin{array}{l}\text { Volume testicular elipsóide } \\
\qquad\left(\mathrm{cm}^{3}\right)\end{array}$ & $6,4 \pm 3,7$ & $0,6-13,6$ & $6,4 \pm 3,4$ & $0,5-12,2$ & $6,4 \pm 3,4$ & $0,6-12,5$ \\
\hline $\begin{array}{l}\text { Volume testicular por } \\
\text { Lambert }\left(\mathrm{cm}^{3}\right)\end{array}$ & $0,009 \pm 0,005$ & $0,001-0,0019$ & $0,009 \pm 0,005$ & $0,001-0,017$ & $0,009 \pm 0,005$ & $0,001-0,017$ \\
\hline Comprimento $(\mathrm{cm})$ & $3,4 \pm 0,8$ & $1,5-4,3$ & $3,4 \pm 0,7$ & $1,5-4,2$ & & \\
\hline Largura $(\mathrm{cm})$ & $2,1 \pm 0,5$ & $1,0-2,9$ & $2,0 \pm 0,5$ & $0,9-2,8$ & & \\
\hline Espessura $(\mathrm{cm})$ & $1,5 \pm 0,4$ & $0,7-2,1$ & $1,6 \pm 0,4$ & $0,6-2,0$ & & \\
\hline Índice de atrofia & & & & & $-6,6 \pm 34,6$ & $-153,6-22,6$ \\
\hline \multicolumn{7}{|l|}{ GRUPOCONTROLE } \\
\hline $\begin{array}{l}\text { Volume testicular elipsóide } \\
\left(\mathrm{cm}^{3}\right)\end{array}$ & $9,7 \pm 2,4$ & $6,6-14,3$ & $9,6 \pm 2,6$ & $4,7-13,7$ & $9,6 \pm 2,4$ & $5,6-14,0$ \\
\hline $\begin{array}{l}\text { Volume testicular por } \\
\text { Lambert }\left(\mathrm{cm}^{3}\right)\end{array}$ & $0,013 \pm 0,003$ & $0,009-0,020$ & $0,013 \pm 0,004$ & $0,006-0,019$ & $0,013 \pm 0,003$ & $0,008-0,019$ \\
\hline Comprimento $(\mathrm{cm})$ & $3,9 \pm 0,3$ & $3,5-4,4$ & $3,8 \pm 0,3$ & $3,2-4,3$ & & \\
\hline Largura $(\mathrm{cm})$ & $2,5 \pm 0,2$ & $2,0-2,9$ & $2,4 \pm 0,3$ & $2,0-3,0$ & & \\
\hline Espessura (cm) & $1,9 \pm 0,3$ & $1,2-2,4$ & $1,9 \pm 0,3$ & $1,3-2,4$ & & \\
\hline Índice de atrofia & & & & & $0,3 \pm 16,9$ & $-35,4-27,8$ \\
\hline
\end{tabular}

A incidência de varicocele foi igual a $61,5 \%$ no grupo afetado e $40 \%$ no grupo controle, sem diferença estatística entre os grupos (x $2=1,77 ;$ g. $1 .=1 ; p=0,18$ $3 \mathrm{p}$ Fisher=0,16), acometendo predominantemente o testículo esquerdo, em ambos os grupos. A varicocele do testículo direito ocorreu exclusivamente em dois pacientes do grupo afetado, sob forma sub-clínica.
Em ambos os grupos a presença de atrofia testicular não estava associada à presença de varicocele, avaliada através do refluxo venoso ( $\mathrm{p}$ Fisher=0,32). Entretanto, detectou-se que o risco de atrofia testicular, na ausência de varicocele, do grupo afetado foi o dobro daquele para o grupo controle (risco igual a 1,92 para o grupo afetado contra 0,81 para o grupo controle), Tabela 2 .

TABELA 2 - Correlação entre atrofia testicular e refluxo venoso - Grupo afetado/Grupo controle.

\begin{tabular}{ccccccc}
\hline $\begin{array}{c}\text { ATROFIA } \\
\text { TESTICULAR }\end{array}$ & \multicolumn{2}{c}{$\begin{array}{c}\text { GRUPOAFETADO } \\
\text { REFLUXO }\end{array}$} & \multicolumn{2}{c}{$\begin{array}{c}\text { GRUPO CONTROLE } \\
\text { REFLUXO }\end{array}$} \\
\hline & Presente & Ausente & Total & Presente & Ausente & Total \\
\hline Presente & 1 & 2 & 3 & 1 & 1 & 2 \\
\hline Ausente & 15 & 8 & 23 & 5 & 8 & 13 \\
\hline Total & 16 & 10 & 26 & 6 & 9 & 15 \\
\hline
\end{tabular}

\section{Discussão}

Embora programas especiais de saúde pública tenham sido implementados na tentativa de erradicação da esquistossomose, essa doença, na sua forma grave, ainda persiste acometendo crianças, adolescentes e adultos jovens diminuindo a produtividade e afetando a qualidade de vida desses pacientes. Os tratamentos clínicos e, nos casos mais severos, os tratamentos cirúrgicos têm produzido progressivamente melhores resultados diminuindo de forma sensível a morbidade e a mortalidade da doença ${ }^{2,5}$. Todavia, a doença grave quando acomete as crianças, como nos pacientes do presente estudo, deixa estigmas graves em vários 
sistemas orgânicos incluindo alterações no desenvolvimento somático e genital ${ }^{13}$.

Os pacientes afetados embora tenham melhorado o desenvolvimento somático, anteriormente descrito ${ }^{3}$, persistem com déficit significativo no desenvolvimento da genitália.

O achado de $61,5 \%$ de varicocele nos pacientes afetados é mais do que quatro vezes a esperada para adolescentes e adultos jovens sem hipertensão porta na mesma faixa etária ${ }^{10,11}$. Verifica-se também uma alta prevalência de varicocele no grupo controle (40\%), podendo sugerir que pode haver algum fator relacionado aos fatores epidemiológicos locais que não estão relacionados à esquistossomose mansônica.

Os volumes testiculares mostraram-se bastante reduzidos nos dois grupos estudados, sugerindo um padrão local próprio, conseqüente ou não a determinados fatores, provavelmente ainda não identificados ou mesmo ainda não estudados. Entretanto se constatou que os volumes testiculares médios do grupo afetado foram significativamente menores que os do grupo controle, o que faz crer que esquistossomose mansônica na forma hepatoesplênica contibui para o hipodesenvolvimento testicular.

Surpreendendo as expectativas, a atrofia testicular não se associou à varicocele, o que leva a pensar que, se não tratada, a varicocele poderá levar ao agravamento da atrofia testicular já existente. Ou então, que a varicocele não provoca atrofia testicular, divergindo do que freqüentemente é citado na literatura ${ }^{8,9,13}$.

Como é amplamente relatado a relação entre varicocele, volume testicular diminuído e infertilidade, estamos avaliando a capacidade reprodutiva dos pacientes que apresentaram as respectivas alterações morfológicas através de espermogramas e dosagens hormonais.

Pretendemos investigar um número maior de adolescentes e adultos jovens com e sem esquistossomose, com as mesmas e outras características epidemiológicas e em outras micro regiões, com o objetivo de definir a verdadeira incidência de varicocele no nosso meio e poder avançar em um melhor esclarecimento da relação que existe entre o refluxo da veia espermática com hipertensão portal, com atrofia testicular e com infertilidade.

\section{Conclusões}

1. Não existe diferença entre a incidência de varicocele do grupo afetado e o grupo controle.

2. É alta a incidência de atrofia testicular em ambos os grupos, mas não está diretamente relacionada à varicocele.

\section{Referências}

1. Coutinho A, Domingues ALC. Esquistossomose mansônica. In: Dani R, Castro LP. Gastroenterologia clínica. Guanabara Koogan: Rio de Janeiro; 1993. p 1697-728.

2. Brandt CT, Maciel DT, Frei Caneca AO, Alexandre F. Hipertensão portal esquistossomótica na criança: avaliação crítica. An Fac Med Univ Fed Pernam 1995;40: 106-10.

3. Brandt CT, Maciel DT, Alexandre AS. Esquistossomose hepatoesplênica em crianças: desenvolvimento pondo-estatural após tratamento cirúrgico. An Fac Med Univ Fed Pernam 1995; 40: 56-60.

4. Brandt CT, Maciel DJS, Frei Caneca AO. Splenectomy associated with ligature of the left gastric vein: analysis of the hepatic functional reserve. Acta Cir Bras 1993; 8: 113-7.

5. Brandt CT, Maciel DT, Frei Caneca AO. Esplenose associada ao tratamento cirúrgico da hipertensão porta esquistossomótica na criança: avaliação de dez anos. An Fac Med Univ Fed Pernam 1999; 44: 15-20.

6. Brandt CT, Maciel DT, Almeida Filho P, Torres IAA. Cintilografia renal em crianças com esquistossomose mansônica, na forma hepatoesplênica, submetidas a esplenectomia, ligadura da veia gástrica esquerda e autoimplante esplênico. An Fac Med Univ Fed Pernam 1998; 43: 13-22.

7. McGee JOD, Grases P. Cirrhosis and portal hypertension: pathological aspects. In: Blumgart LH. Surgery of the liver and the billiary tract. Edinburgh: Churchill Livingstone; 1988. p 1301-12.

8. Chatel A, Bigot JM, Dectot H, Helenon C. Radiological anatomy od the spermatic veins: report of 152 retrograde spermatic phlebographies. J Chir 1978; 115: 443-50.

9. Chatel A, Bigot JM, Dectot H, Helenon C. The collateral circulations of spermatic veins. J Radiol 1979; 60: 121-7.

10. Sayfan J, Soffer Y, Mannor H, Witz E, Orda R Varicocele in youth: a therapeutic dilemma. Ann Surg 1988; 207: 223-7.

11. Rozanski TA, Bloom DA. Male genital tract. In: Oldham KT, Colombani PM, Foglia RP. Surgery of infants and children: scientific principles and practice. Philadelphia: Lippincott Raven Publishers; 1997. p 65-9.

12. Burringtonu RS. Handbook of mathematical tables and formulas. 5ed. McGraw-Hill; 1973. p 27.

13. Belloli G, D'agostino S, Pesce C, Fantuz E. Varicocele in childhood and adolescence and other testicular anomalies: a epidemiological study. Pediatr Med Chir 1993;15(2): 159-62. 
Brandt F T e col.

Brandt FT, Albuquerque CDC, Brandt CT, Barbosa CD. Varicocele and testicular volume in adolescents and young adults with surgical hepatosplenic schistosomiasis. Acta Cir Bras [serial online] 2003 Jul-Aug;18(4). Available from URL: http://www.scielo.br/acb.

ABSTRACT - Purpose: This case-control study was aimed at evaluating testicular volume, puberal and genital development, and the prevalence of varicocele in adolescents and young adults with shistosomiasis in the hepatosplenic form associated with bleeding esophageal varices. Methods: The study involved 22 young males with schistosomiasis as described, submitted to splenectomy, left gastric vein ligature and auto-implant of splenic tissue in the greater omentum during childhood. The mean follow-up period was 4 years after surgery. The control group included healthy volunteers from a school in the same region where the cases lived. They were randomly selected, matched by age and epidemiological characteristics, and had schistosomiasis ruled out. All participants had complete history taken and were submitted to a thorough clinical and ultrasonographic examinations. Special attention was given to sexual characteristics, testicular ultrasonography and dopplerfluxometry of spermatic veins. Results and Conclusions: Patients with schistosomiasis had significant deficits in puberal and genital development, testicular volume, specially the left one, and a high prevalence of varicocele $(61.5 \%)$ without a straight association with testicular atrophy.

KEY WORDS - Varicocele. Testicular volume. Schistosomiasis mansoni. Child. Adolescent. Young adult. Portal hypertension.

Correspondência:

Conflito de interesse: nenhum

Fonte de financiamento: nenhuma

Prof. Frederico Teixeira Brandt

R. Afonso Celso, 142/301

52060-110 Recife - PE

ftbrandt@elogica.com.br

Data do recebimento: 04/03/2003

Data da revisão: $13 / 03 / 2003$

Data da aprovação: 28/03/2003 